\title{
Low-Energy Electron Emission in the Strong-Field Ionization of Rare Gas Clusters
}

\author{
Bernd Schütte, ${ }^{1, *}$ Christian Peltz, ${ }^{2}$ Dane R. Austin, ${ }^{1}$ Christian Strüber, ${ }^{1}$ Peng Ye, ${ }^{1}$ Arnaud Rouzée, ${ }^{3}$ \\ Marc J. J. Vrakking, ${ }^{3}$ Nikolay Golubev, ${ }^{4}$ Alexander I. Kuleff, ${ }^{4,5}$ Thomas Fennel, ${ }^{2,3, \dagger}$ and Jon P. Marangos, ${ }^{1, \$}$ \\ ${ }^{1}$ Department of Physics, Imperial College London, South Kensington Campus, SW7 2AZ London, United Kingdom \\ ${ }^{2}$ Institute of Physics, University of Rostock, Albert-Einstein-Strasse 23, 18059 Rostock, Germany \\ ${ }^{3}$ Max-Born-Institut, Max-Born-Strasse 2A, 12489 Berlin, Germany \\ ${ }^{4}$ Theoretische Chemie, PCI, Universität Heidelberg, Im Neuenheimer Feld 229, 69120 Heidelberg, Germany \\ ${ }^{5}$ ELI-ALPS, Budapesti út 5, H-6728 Szeged, Hungary
}

(Received 5 April 2018; published 8 August 2018)

\begin{abstract}
Clusters and nanoparticles have been widely investigated to determine how plasmonic near fields influence the strong-field induced energetic electron emission from finite systems. We focus on the contrary, i.e., the slow electrons, and discuss a hitherto unidentified low-energy structure (LES) in the photoemission spectra of rare gas clusters in intense near-infrared laser pulses. For $\mathrm{Ar}$ and $\mathrm{Kr}$ clusters we find, besides field-driven fast electrons, a robust and nearly isotropic emission of electrons with $<4 \mathrm{eV}$ kinetic energies that dominates the total yield. Molecular dynamics simulations reveal a correlated fewbody decay process involving quasifree electrons and multiply excited ions in the nonequilibrium nanoplasma that results in a dominant LES feature. Our results indicate that the LES emission occurs after significant nanoplasma expansion, and that it is a generic phenomenon in intense laser nanoparticle interactions, which is likely to influence the formation of highly charged ions.
\end{abstract}

DOI: 10.1103/PhysRevLett.121.063202

Strong-field ionization of atoms and molecules has been studied for several decades [1-4], and the fundamental processes are often considered to be well understood. In particular, the picture of electron release by tunneling followed by the competition among direct emission, recombination, and field-driven elastic scattering provides a powerful concept to understand central features such as characteristic energy cutoffs in atomic and molecular high-order above-threshold ionization spectra [5] and highharmonic generation [6]. Nevertheless, surprising observations are still being made, even in these comparatively simple systems. Recent discoveries include the holographic nature of low-energy features in photoelectron spectra [7] and forklike patterns associated with late returns [8], as well as a pronounced spikelike low-energy structure (LES) that becomes particularly pronounced in intense midinfrared fields $[9,10]$. In these features, field-driven rescattering in the Coulomb field of the residual ion plays a prominent role [7,11-13].

Clusters and nanoparticles in intense laser fields provide a versatile platform to explore cooperative and finite-size effects such as avalanching-induced nanoplasma formation and field localization, and their implications for energetic ion, photon, and electron emission from nanostructures $[14,15]$. Backscattering processes similar to the atomic and molecular case are observed in the locally enhanced near fields and, for extremely short pulses, can be controlled with the field waveform $[16,17]$. Strong absorption $[18]$ and formation of highly charged ions $[19,20]$ and particularly fast electrons can be realized for transient plasmonic resonance enhancement in expanded cluster nanoplasmas [21-23], where forward rescattering can even become the leading field-driven electron acceleration mechanism [24,25].

Surprisingly, slow and weakly bound electrons are at least similarly important, as they are, e.g., pivotal for explaining the ion spectra from laser-driven clusters [26]. In excitations of clusters with intense femtosecond laser fields, the majority of cluster electrons remains trapped in the cluster Coulomb potential during illumination [14], but they must escape eventually to rationalize the observed dominance of highly charged ion emission [27]. Moreover, slow electrons are of crucial importance in the interactions of lasers with human tissue, where they induce tissue damage via breakup of DNA [28]. So far, however, little is known about the production of slow electrons in intense laser-cluster interactions and the underlying physics responsible for their generation, although such information is essential to understanding and harnessing laser-driven nanoplasmas.

In this Letter we aim at resolving these mechanisms and report the observation of a hitherto unreported, dominant, and robust low-energy feature in electron spectra resulting from the exposure from rare gas clusters to ultrashort nearinfrared (NIR) pulses. We find that the mechanism behind the low-energy structure from clusters is different from that for atoms and small molecules. In particular, the nearly isotropic distribution of LES electrons suggests a delayed emission resulting from a relaxation process. Our microscopic molecular dynamics analysis reproduces the LES 
feature qualitatively and identifies a few-body decay mechanism that is based on collisional energy transfer from multiply excited ions to delocalized cluster electrons, leading to slow autoionization of the cluster. The fact that the yield of the LES emission can exceed the prompt, fieldinduced emission achieved during illumination signifies the general importance of autoionization processes of nanoparticles exposed to strong laser fields.

The experimental setup was previously described [29]. Briefly, a Ti:sapphire laser system provided $30 \mathrm{fs}$ pulses (FWHM), at $800 \mathrm{~nm}$ with up to $8 \mathrm{~mJ}$ pulse energy. After passing a $\lambda / 2$ wave plate for polarization control, the pulses were focused by a spherical silver mirror $(15 \mathrm{~cm}$ focal length) and intersected a pulsed jet of clusters. The backing pressure was changed between 2.5 and 6 bar, and the Hagena scaling law was used to estimate the corresponding average cluster size [30]. An orifice with $0.5 \mathrm{~mm}$ diameter was used to form a narrow cluster beam. To avoid that Rydberg atoms and ions emerging from the cluster expansion produce a spurious signal due to field ionization via detector electric fields [26,31], we used a field-free time-offlight (TOF) electron spectrometer with a small $\left(<3^{\circ}\right)$ acceptance angle [32]. The detection efficiency of slow electrons was increased by a grid in front of the microchannel plate detector that accelerated all incoming electrons by $500 \mathrm{eV}$ (at the same time this prevents ions from being detected).

Figure 1(a) displays typical electron TOF spectra from $\operatorname{Ar}_{N} \quad(\langle N\rangle=4000)$ for pulses polarized parallel and perpendicular to the TOF spectrometer axis. The corresponding electron kinetic energy spectra [Fig. 1(b)] show three main features that are analyzed in more detail below. First, the spectra contain an unstructured high-energy contribution that decays nearly exponentially $\left[Y(E) \propto \exp ^{-E / E_{\text {decay }}}\right]$ with a decay constant of $E_{\text {decay }}=55 \mathrm{eV}$ for emission parallel to the laser polarization (dashed line), which is consistent with previous results [33]. The strongly enhanced yield of these "fast" electrons along the polarization axis indicates fielddriven emission [21,22,24,29,34,35].

Second, a plateaulike contribution is observed for both $\mathrm{Ar}$ and $\mathrm{Kr}$ clusters that shows a shoulder between about 10 and $15 \mathrm{eV}$. The shoulder was previously attributed to correlated electronic decay processes [36] and cascades of intra-Rydberg interatomic Coulombic decay transitions [37,38] producing singly charged ions and electrons with kinetic energies up to the ionization potential of the cluster atoms, i.e., up to about $15.8 \mathrm{eV}$ for Ar. Further examples of recently discovered correlation-driven cluster ionization processes include collective autoionization [39], autoionization of spin-orbit-excited states [40], as well as the relaxation of multiply charged ions by Auger decay [41] and autoionization [42].

Most importantly, the spectra exhibit an additional exponentially decaying low-energy structure from slow electrons with kinetic energies $<4 \mathrm{eV}$, that, in contrast to
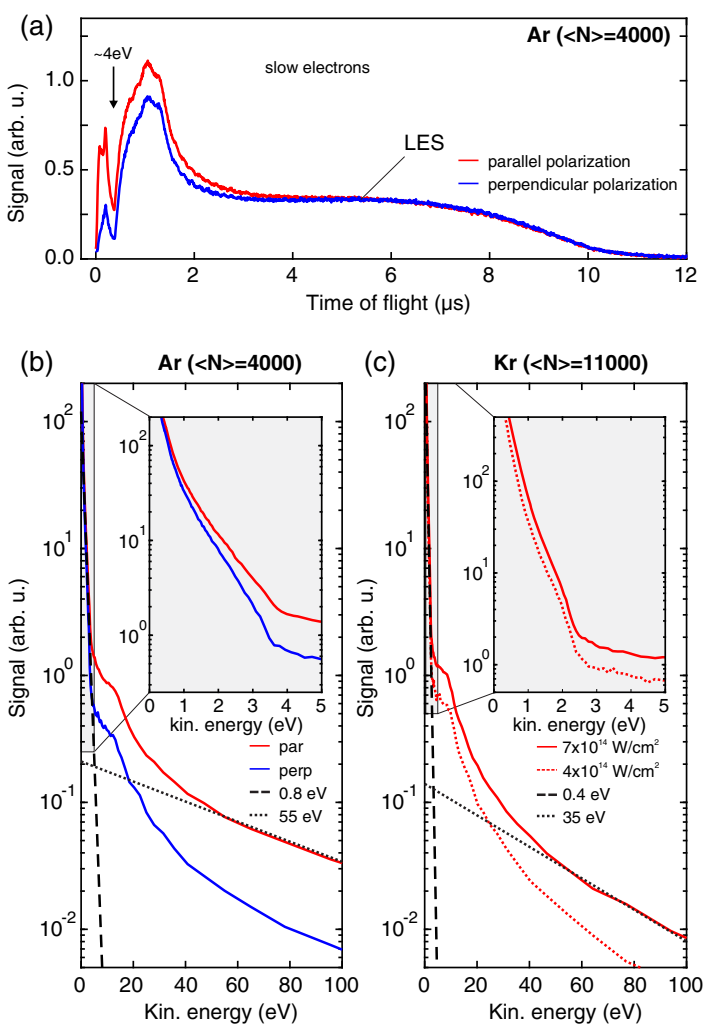

FIG. 1. (a) Electron TOF spectra from $\operatorname{Ar}_{N}$ clusters $(\langle N\rangle=4000)$ after excitation with 30 fs NIR laser pulses $\left(I=7 \times 10^{14} \mathrm{~W} / \mathrm{cm}^{2}\right)$ with parallel and perpendicular polarization with respect to the TOF spectrometer axis. The spectra are dominated by a low-energy structure (LES) with kinetic energies $<4 \mathrm{eV}$. (b) Corresponding electron kinetic energy spectra on a logarithmic scale. Below $4 \mathrm{eV}$, the electron kinetic energy distribution can be approximated by an exponential function with a decay constant of $0.8 \mathrm{eV}$. (Inset) Enlargement of the LES. (c) Electron spectra from $\mathrm{Kr}_{N}$ recorded at two different intensities for parallel polarization.

the fast electron feature, is only weakly polarization dependent; see the inset of Fig. 1(b). This nearly isotropic emission behavior differs qualitatively from the laser-aligned character of the LES reported for atoms and molecules [9-12]. We emphasize that the application of retarding voltages in the TOF measurement, as often used in cluster experiments $[21,22,29,34]$, would prevent the observation of the lowenergy electrons, confirmed when we applied a small retarding voltage to the electrons. The LES remains a robust feature when varying cluster size, laser intensity, and the cluster constituents; see Fig. 1(c). This observation supports its general significance for intense laser-cluster interactions and strongly motivates its clarification.

Since a complete time-dependent correlated quantum description is out of reach, we performed molecular dynamics (MD) simulations to explore the origin of the low-energy electron emission [26]. The dynamics of atoms, ions, and plasma electrons is described classically using a regularized Coulomb potential and including the laser 
field in the dipole approximation. The neglect of magnetic field effects and pulse propagation is well justified in the considered parameter regime [43]. Dispersive interatomic forces are treated via a binary Lennard-Jones interaction and the clusters are initialized as relaxed icosahedra. Atomic ionization events due to tunneling and electron impact are modeled via Monte Carlo sampling using effective quantum-mechanical rates, including local-field induced ionization potential depression [26]. Note that atomic autoionization [40,41] and many-body recombination processes in the nanoplasma, which dominantly couple to highly excited states [44], are only qualitatively included in the correlated classical treatment.

The MD results obtained for $\mathrm{Ar}_{5083}$ using parameters similar to the experiment reproduce two of the most prominent features of the measured electron spectra and show two exponential contributions with strongly differing decay constants; see Fig. 2(a). While the laser-aligned highenergy feature (decay constant of $75 \mathrm{eV}$ ) is present already when evaluating single-particle energy spectra of emitted electrons immediately after the laser pulse (red spectrum), the cluster dynamics must be calculated for several picoseconds before the pronounced and isotropic LES feature (decay constant of $2 \mathrm{eV}$ ) emerges in the distribution (blue spectrum). This behavior confirms that the origin of the LES is not field-driven emission but a slow relaxation process. Note that the decay constant of the high-energy feature is larger than in the experiment because of focal volume averaging that was not taken into account in the simulation. The decay constant of the simulated LES agrees qualitatively with the experiment, but electron emission will take place over much longer timescales $[40,42]$ than those for which calculations are currently feasible, so exact agreement is not expected.

A closer analysis uncovers the emission dynamics. Nanoplasma ignition by tunneling during laser excitation triggers a collisional ionization avalanche [see Fig. 2(b)], leading to a high average inner ionization stage $q_{i i} \gtrsim 6$ (blue curve). This nonresonant excitation leads to modest absorption (about $300 \mathrm{eV}$ per atom) and initiates cluster expansion (green), but it induces only relatively modest field-driven outer ionization to the continuum $\left(q_{o i}<0.2\right.$, black curve). The evolution map of the single-particle energy spectra of activated electrons in Fig. 2(c) displays a rapid emergence of the fast electrons during illumination and the formation of a very deep $(>0.5 \mathrm{keV})$ cluster potential that flattens quickly due to cluster expansion [45]. At the end of the phase shown in Figs. 2(b) and 2(c), i.e., $150 \mathrm{fs}$ after the peak of the laser pulse, only the fielddriven fast emission has been completed, while most $(>97 \%)$ activated electrons remain trapped.

The LES emission is captured in the long-term emission dynamics in Fig. 2(d). Whereas the fast electron contribution $(E \geq 4 \mathrm{eV}$, red) remains effectively unchanged on
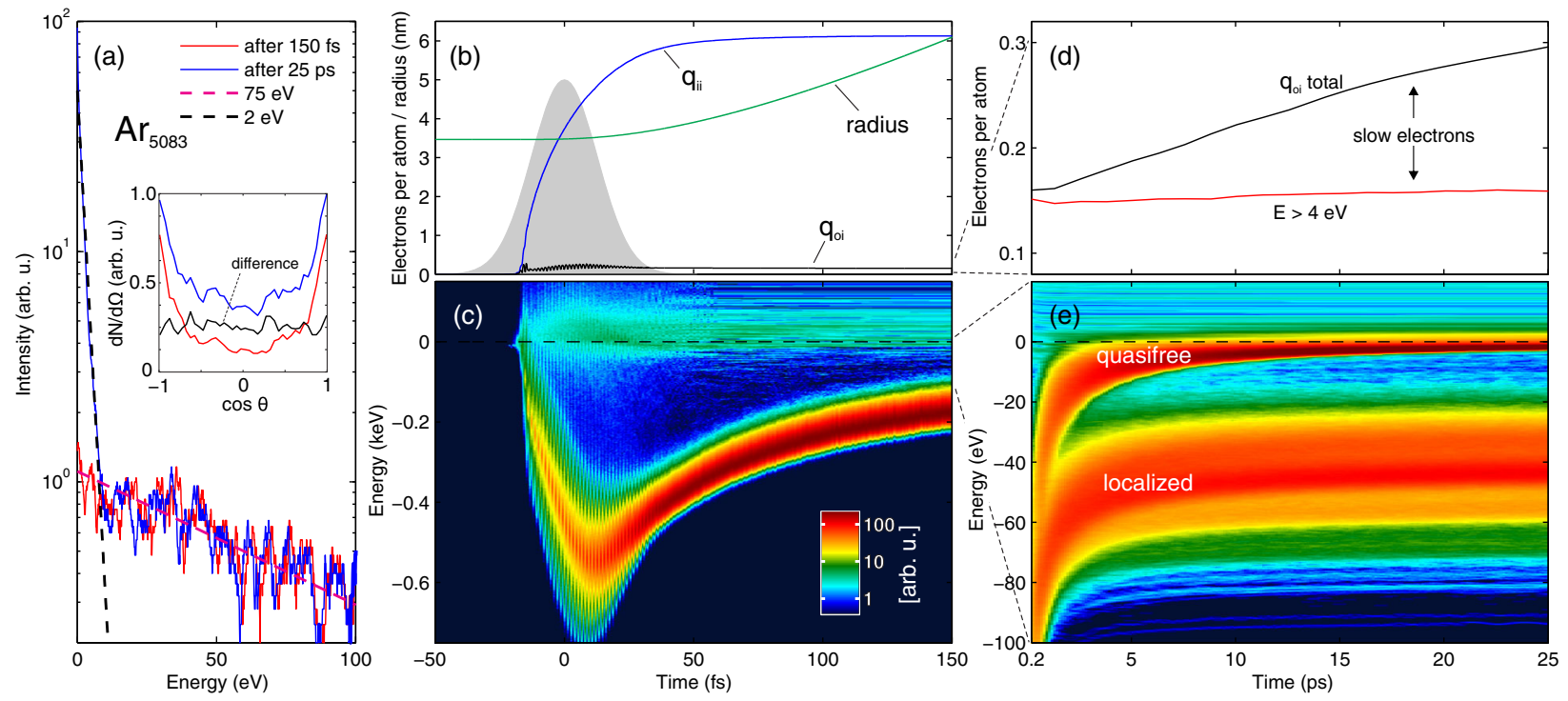

FIG. 2. MD results for $\mathrm{Ar}_{5083}$ excited with a $30 \mathrm{fs}$ pulse at a central wavelength of $800 \mathrm{~nm}$ and a peak intensity of $I=7 \times 10^{14} \mathrm{~W} / \mathrm{cm}^{2}$. (a) Electron energy spectra as extracted from single-particle energy distributions shortly after illumination (red) and at $t=25 \mathrm{ps}$ after the pulse peak (blue). The dashed lines are guides for the eye and reflect exponential decays (decay constants as indicated). (Inset) Angular electron distributions shown with respect to the laser polarization. Clearly, the LES is only reproduced in the simulations if these are continued for several picoseconds after the peak of the laser pulse. (b) Evolution of the inner and outer ionization $\left(q_{i i}\right.$ and $\left.q_{o i}\right)$ per atom (blue and black curves) and the cluster radius (blue) on femtosecond timescales. The laser intensity envelope is illustrated by the gray area. (c) Corresponding evolution of the single-particle energy distribution of electrons that have been set free from their parent atom in a tunnel ionization or electron impact ionization process. (d) Evolution of the total outer ionization (black) and the yield of fast electrons with $E>4 \mathrm{eV}$ (red) that are produced per cluster atom on a picosecond timescale. (e) Corresponding evolution of the single-particle energy distribution. 
picosecond timescales, the increase of the total yield by $\approx 700$ electrons (black) reflects the emission of slow electrons, which become the dominant contribution to the electron energy distribution after about $25 \mathrm{ps}$.

To identify the microscopic process underlying the LES emission, we analyzed the long-term evolution of the single-particle spectra, which show a splitting of the bound population into two bands after about 1 ps; see Fig. 2(e). While the feature at lower energy reflects recombined electrons that are localized in ground and excited states at individual ions, the weakly bound component corresponds to delocalized (quasifree) electrons that have become decoupled from the localized electrons during expansion [46]. The formation of the low-energy feature at $E \gtrsim 0$ coincides with the convergence of the quasifree electron population towards the continuum edge, indicating a link between the quasifree and LES electrons. Nevertheless, it should be emphasized that the electrons are not simply released after expansion sufficient for the cluster potential to become shallow. As Fig. 3(e) shows, the quasifree electron peak becomes sharper due to expansion cooling, preventing a direct release from the potential [26]. Hence, so far, the analysis indicates a connection of the LES electrons to the existence of quasifree electrons in the expanding cluster, but it does not yet rationalize their final continuum energy or the mechanism by which this continuum energy is acquired.

The trajectories of individual escaping electrons reveal the nature of the low-energy electron emission. As a representative set, we analyzed seven electrons emitted after $t=12.5 \mathrm{ps}$ in a $250 \mathrm{fs}$ time interval [Fig. 3(a)], defining the moment when electrons are finally promoted to the continuum as the escape time $t_{\text {esc }}$. Rapid jumps (durations $\lesssim 100$ as) of the single-particle energies $E_{\mathrm{sp}}(t)$ from values in the spectral range of quasifree electrons to the continuum are observed for all trajectories around $t_{\text {esc }}$ [Fig. 3(c)]. Combined with the synchronized passage of a nearby ion [Fig. 3(b)], the trajectory analysis evidences an emission induced by single collision events of delocalized electrons with individual ions that, as a result of recombination processes, carry one or more excited electrons with them. The detailed energetics of one selected LES electron (blue trajectory) illustrate the mechanism of the energy transfer. The electron's energy capture proceeds in a collision with an ion $(q=6)$ surrounded by four orbiting localized electrons [Fig. 3(d)]. The corresponding singleparticle energy evolutions show that all four localized electrons interact strongly with the colliding electron [Fig. 3(e)], so no single localized electron can be identified as the dominant energy exchange partner. This fact underlines the few-body nature of the collision process. However, the energy associated with the subsystem of the multiply excited ion (i.e., the sum of the kinetic energies and mutual interaction energies of the ion core and the associated localized excited electrons) shows that the energy gain of the emitted electron is provided completely

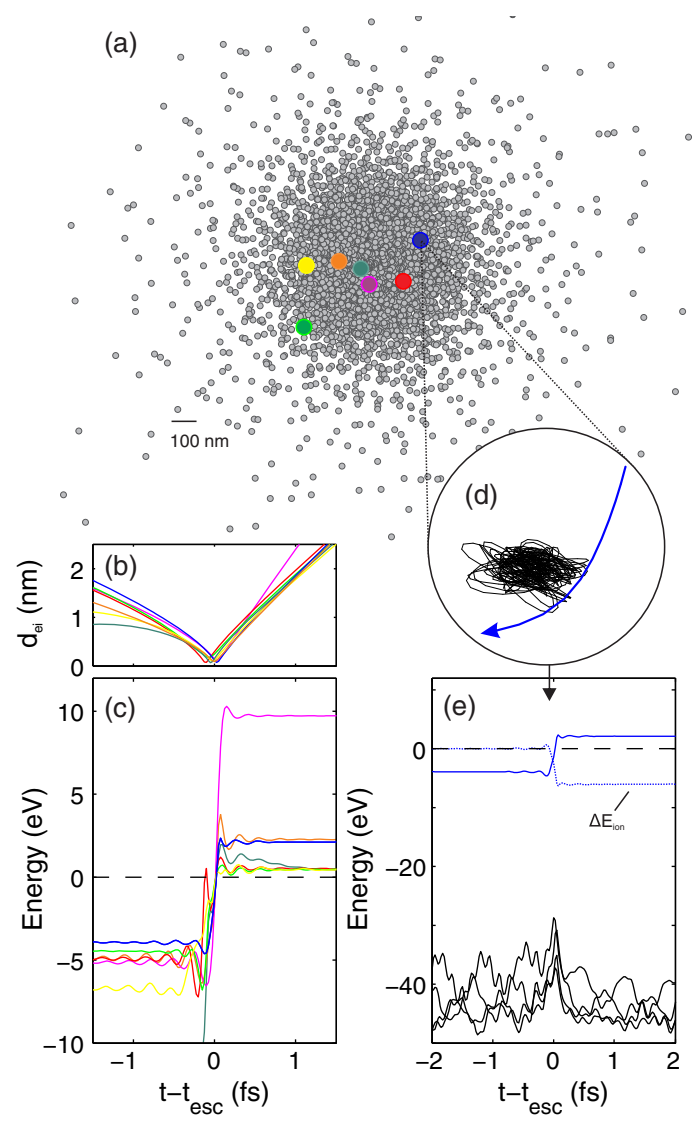

FIG. 3. (a) Snapshot of the cluster at $t=12.5 \mathrm{ps}$ (gray dots denote atoms) and position of the seven selected electrons that escape in the subsequent $250 \mathrm{fs}$ (colored patches). (b) Evolution of the distance $d_{\mathrm{ei}}$ to the nearest ion and (c) the single-particle energies $E_{\mathrm{sp}}$ for the individual electron trajectories around the moment of escape $t_{\mathrm{esc}}$, characterizing the bound-continuum transition via the last change from negative to positive of $E_{\mathrm{sp}}$. (d) Trajectories of a selected LES electron during emission (blue) and of the four localized electrons (black) associated with the encountered ion. (e) Corresponding single-particle energy evolutions (solid blue and black lines) and change of the energy $E_{\text {ion }}$ associated with the subsystem of the multiply excited ion (blue dashed line).

by the multiply excited ion (see the dashed blue curve). Note that the one-to-one correspondence between LES electron energy gain and ion energy loss is to be expected from the fact that in the expanding cluster the average interatomic distance has significantly increased (see Fig. 2). The same picture applies to all LES electrons highlighted in Fig. 3(a). Our analysis thus supports that collisional energy transfer from multiply excited ions to near-threshold electrons results in substantial LES electron emission.

In contrast to the strong-field ionization of atoms, where the LES was suppressed for circularly polarized light [9], we expect the LES to remain dominant in the strong-field ionization of clusters using circularly polarized light since the polarization has little overall influence on the ionization dynamics [47]. In addition to the described collisional 
process, other correlated electronic processes may contribute to the observed cluster LES and may take place up to the nanosecond timescale. The delayed emission of slow electrons either precedes, and thereby prevents, electronion recombination (as in the collisional process discussed here) or occurs after recombination (as, e.g., in atomic autoionization), thus providing a possible explanation for the observation of highly charged ions in strong-field ionization of clusters $[19,20]$. Further investigation of the contributions of these channels by treatments beyond atomistic molecular dynamics will be needed to fully understand their role.

In summary, we report in this Letter the observation of a thus far unidentified and surprisingly strong emission of low-energy electrons from intense laser-cluster interactions. Our analysis shows that this emission is robust and generic, and that it can be attributed to autoionization of the excited cluster nanoplasma and is thus decoupled from the prompt, field-driven emission during laser excitation. Microscopic quasiclassical simulations reproduce the experimental features qualitatively and suggest that few-body collisional energy transfer from multiply excited ions to quasifree electrons contributes to the observation of the LES emission. A complete description of the LES would require a fully correlated quantum treatment of the cluster and the multiply excited ions, which is currently out of reach. Meanwhile, time-resolved experiments with terahertz pulses combined with semiclassical simulations would be ideal to trace the evolution leading to the observed asymptotic state. The present findings highlight the importance of correlated relaxation phenomena in laserdriven nanostructures and their applications.

We gratefully acknowledge financial support from the Deutsche Forschungsgemeinschaft (DFG) via Grants No. SFB652 and No. SPP1840, a Heisenberg fellowship (T. F.), and a research fellowship (B. S.). J. P. M., D. R. A., C. S., and P. Y. would like to acknowledge funding from Engineering and Physical Sciences Research Council (EPSRC) (Grant No. EP/313 I032517/1), European Research Council (ERC) (ASTEX Project No. 290467) and the EPSRC/Defence Science and Technology Laboratory (DSTL) Multidisciplinary University Research Initiative (MURI) Grant No. EP/N018680/1. Computing time provided by the Norddeutscher Verbund für Hoch- und Höchstleistungsrechnen (HLRN) (Project ID No. mvp00011) is gratefully acknowledged.

*bschutte@imperial.ac.uk

†thomas.fennel@uni-rostock.de

*j.marangos@imperial.ac.uk

[1] P. Agostini, F. Fabre, G. Mainfray, G. Petite, and N. K. Rahman, Phys. Rev. Lett. 42, 1127 (1979).

[2] P. B. Corkum, Phys. Rev. Lett. 71, 1994 (1993).
[3] W. Becker, F. Grasbon, R. Kopold, D. Milošević, G. Paulus, and H. Walther, Adv. At. Mol. Opt. Phys. 48, 35 (2002).

[4] W. Becker, X. Liu, P. J. Ho, and J. H. Eberly, Rev. Mod. Phys. 84, 1011 (2012).

[5] D. B. Milošević, G. G. Paulus, D. Bauer, and W. Becker, J. Phys. B 39, R203 (2006).

[6] M. Lewenstein, P. Balcou, M. Y. Ivanov, A. L'Huillier, and P. B. Corkum, Phys. Rev. A 49, 2117 (1994).

[7] Y. Huismans, A. Rouzée, A. Gijsbertsen, J. Jungmann, A. Smolkowska, P. Logman, F. Lepine, C. Cauchy, S. Zamith, T. Marchenko et al., Science 331, 61 (2011).

[8] D. D. Hickstein, P. Ranitovic, S. Witte, X.-M. Tong, Y. Huismans, P. Arpin, X. Zhou, K. E. Keister, C. W. Hogle, B. Zhang, C. Ding, P. Johnsson, N. Toshima, M. J. J. Vrakking, M. M. Murnane, and H. C. Kapteyn, Phys. Rev. Lett. 109, 073004 (2012).

[9] C. I. Blaga, F. Catoire, P. Colosimo, G. G. Paulus, H. G. Muller, P. Agostini, and L. F. DiMauro, Nat. Phys. 5, 335 (2009).

[10] W. Quan, Z. Lin, M. Wu, H. Kang, H. Liu, X. Liu, J. Chen, J. Liu, X. T. He, S. G. Chen, H. Xiong, L. Guo, H. Xu, Y. Fu, Y. Cheng, and Z.Z. Xu, Phys. Rev. Lett. 103, 093001 (2009).

[11] T.-M. Yan, S. V. Popruzhenko, M. J. J. Vrakking, and D. Bauer, Phys. Rev. Lett. 105, 253002 (2010).

[12] C. Liu and K. Z. Hatsagortsyan, Phys. Rev. Lett. 105, 113003 (2010).

[13] A. Kästner, U. Saalmann, and J. M. Rost, Phys. Rev. Lett. 108, 033201 (2012).

[14] U. Saalmann, C. Siedschlag, and J. Rost, J. Phys. B 39, R39 (2006).

[15] T. Fennel, K.-H. Meiwes-Broer, J. Tiggesbäumker, P.-G. Reinhard, P. M. Dinh, and E. Suraud, Rev. Mod. Phys. 82, 1793 (2010).

[16] S. Zherebtsov, T. Fennel, J. Plenge, E. Antonsson, I. Znakovskaya, A. Wirth, O. Herrwerth, F. Süßmann, C. Peltz, I. Ahmad et al., Nat. Phys. 7, 656 (2011).

[17] M. Krüger, M. Schenk, and P. Hommelhoff, Nature (London) 475, 78 (2011).

[18] J. Zweiback, T. Ditmire, and M. D. Perry, Phys. Rev. A 59, R3166 (1999).

[19] E. M. Snyder, S. A. Buzza, and A. W. Castleman, Phys. Rev. Lett. 77, 3347 (1996).

[20] T. Ditmire, J. W. G. Tisch, E. Springate, M. B. Mason, N. Hay, R. A. Smith, J. Marangos, and M. H. R. Hutchinson, Nature (London) 386, 54 (1997).

[21] V. Kumarappan, M. Krishnamurthy, and D. Mathur, Phys. Rev. A 66, 033203 (2002).

[22] E. Springate, S. A. Aseyev, S. Zamith, and M. J. J. Vrakking, Phys. Rev. A 68, 053201 (2003).

[23] T. Döppner, T. Fennel, P. Radcliffe, J. Tiggesbäumker, and K.-H. Meiwes-Broer, Phys. Rev. A 73, 031202 (2006).

[24] T. Fennel, T. Döppner, J. Passig, C. H. Schaal, J. Tiggesbäumker, and K.-H. Meiwes-Broer, Phys. Rev. Lett. 98, 143401 (2007).

[25] J. Passig, S.Zherebtsov, R. Irsig, M. Arbeiter, C. Peltz, S. Göde, S. Skruszewicz, K.-H. Meiwes-Broer, J. Tiggesbäumker, M. F. Kling et al., Nat. Commun. 8, 1181 (2017).

[26] T. Fennel, L. Ramunno, and T. Brabec, Phys. Rev. Lett. 99, 233401 (2007). 
[27] T. Döppner, J. P. Müller, A. Przystawik, S. Göde, J. Tiggesbäumker, K.-H. Meiwes-Broer, C. Varin, L. Ramunno, T. Brabec, and T. Fennel, Phys. Rev. Lett. 105, 053401 (2010).

[28] B. Boudaiffa, P. Cloutier, D. Hunting, M. A. Huels, and L. Sanche, Science 287, 1658 (2000).

[29] B. Schütte, S. Patchkovskii, P. Ye, D. R. Austin, C. Brahms, C. Strüber, T. Witting, M. Y. Ivanov, J. W. G. Tisch, and J. P. Marangos, Sci. Rep. 6, 39664 (2016).

[30] O. F. Hagena and W. Obert, J. Chem. Phys. 56, 1793 (1972).

[31] B. Schütte, M. Arbeiter, T. Fennel, M. J. J. Vrakking, and A. Rouzée, Phys. Rev. Lett. 112, 073003 (2014).

[32] O. Hemmers, S. B. Whitfield, P. Glans, H. Wang, D. W. Lindle, R. Wehlitz, and I. A. Sellin, Rev. Sci. Instrum. 69, 3809 (1998).

[33] V. Kumarappan, M. Krishnamurthy, and D. Mathur, Phys. Rev. A 67, 043204 (2003).

[34] Y. L. Shao, T. Ditmire, J. W. G. Tisch, E. Springate, J. P. Marangos, and M. H. R. Hutchinson, Phys. Rev. Lett. 77, 3343 (1996).

[35] J. Passig, R. Irsig, N. X. Truong, T. Fennel, J. Tiggesbäumker, and K. H. Meiwes-Broer, New J. Phys. 14, 085020 (2012).
[36] B. Schütte, M. Arbeiter, T. Fennel, G. Jabbari, A. I. Kuleff, M. J. J. Vrakking, and A. Rouzée, Nat. Commun. 6, 8596 (2015).

[37] K. Nagaya et al., Nat. Commun. 7, 13477 (2016).

[38] D. Iablonskyi et al., Phys. Rev. Lett. 117, 276806 (2016).

[39] Y. Ovcharenko et al., Phys. Rev. Lett. 112, 073401 (2014).

[40] B. Schütte, J. Lahl, T. Oelze, M. Krikunova, M. J. J. Vrakking, and A. Rouzée, Phys. Rev. Lett. 114, 123002 (2015).

[41] B. Schütte, M. J. J. Vrakking, and A. Rouzée, Phys. Rev. A 95, 063417 (2017).

[42] D. Komar, L. Kazak, M. Almassarani, K.-H. Meiwes-Broer, and J. Tiggesbäumker, Phys. Rev. Lett. 120, 133207 (2018).

[43] C. Peltz, C. Varin, T. Brabec, and T. Fennel, New J. Phys. 14, 065011 (2012).

[44] H. A. Bethe and E. E. Salpeter, Quantum Mechanics of One-and Two-Electron Atoms (Springer Science+Business Media, New York, 2012).

[45] U. Saalmann and J. M. Rost, Eur. Phys. J. D 36, 159 (2005).

[46] M. Arbeiter, C. Peltz, and T. Fennel, Phys. Rev. A 89, 043428 (2014).

[47] M. Krishnamurthy, D. Mathur, and V. Kumarappan, Phys. Rev. A 69, 033202 (2004). 